\title{
A CHECK-VALVED SILICONE DIAPHRAGM PUMP
}

\author{
Ellis Meng, Xuan-Qi Wang, Howen Mak, and Yu-Chong Tai \\ Caltech Micromachining Lab, 136-93, Caltech, Pasadena, CA 91126, USA
}

\begin{abstract}
Two generations of check-valved silicone rubber diaphragm pumps are presented. Significant improvements have been made from pump to pump including the design and fabrication of a double-sided check valve, a bossed silicone membrane, and silicone gaskets. Water flow rates of up to $13 \mathrm{ml} / \mathrm{min}$ and a maximum back pressure of $5.9 \mathrm{kPa}$ were achieved through pneumatic operation with an external compressed air source. Using a custom designed solenoid actuator, flow rates of up to $4.5 \mathrm{ml} / \mathrm{min}$ and a maximum back pressure of $2.1 \mathrm{kPa}$ have been demonstrated.
\end{abstract}

\section{INTRODUCTION}

A reciprocating diaphragm with two passive check valves is a common micropump scheme [1-3]. Other schemes include diffuser $[4,5]$, electrohydrodynamic $[6,7]$, rotary [8], and gear pumps [9]. In diaphragm pumps, fluidic transport is achieved by alternating the production of under- and over-pressures in the pump chamber, resulting in a supply mode and a pump mode, respectively. During these modes, pumping occurs only when enough pressure difference is produced to overcome the cracking pressure of the rectifying valves. Since microactuators are limited by a small stroke capability, low cracking pressure, reverse leakage, and flow resistance are highly desirable characteristics in check valves.

Previously, Shoji et al [10] and Smith and Hök [11] demonstrated valves designed for use with micropumps, however, both exhibited some degree of reverse leakage and had a limited flow rate. Parylene check valves modified from [12] satisfied the above requirements with the additional advantage of negligible stiction and surface tension effects. The geometric configuration of the moving membrane element of the check valve, in addition to the low Young's modulus of Parylene $(\sim 2.8$ $\mathrm{GPa}$ ), resulted in high deflections and, thus, negligible flow resistance. In fact, the flow resistance characteristics of the check valve were nearly identical to those of a plain orifice. Pumps designed with these valves were capable of reverse blocking even in the neutral state unlike valveless pumps [4-7].

It is also necessary to minimize the pressure required to deflect the pumping membrane. Since only weak forces are available, a membrane material with low Young's modulus is ideal. Silicone rubber (MRTV1; American Safety Technologies, Inc.) has a low Young's modulus and was implemented as a membrane material in microvalves [13] and micropumps [14] due to its high elongation (100-1000\%). Thus, large deflections and efficient use of pump chamber volume are realized. Silicone rubber (Sylgard 184; Dow Corning) was also used in this pump to create molded gaskets.

The testing apparatus involved the use of Plexiglas pump seats in addition to a combination of gluing and clamping. Actuation was supplied by a custom designed solenoid. For the second generation pump, pneumatic operation with a pressurized air source controlled by a three-way solenoid valve was also examined.

\section{DESIGN AND FABRICATION}

The first generation pump was assembled by gluing chips together (Fig. 1). As this made it difficult to assemble and repair, the second generation (Fig. 2) was

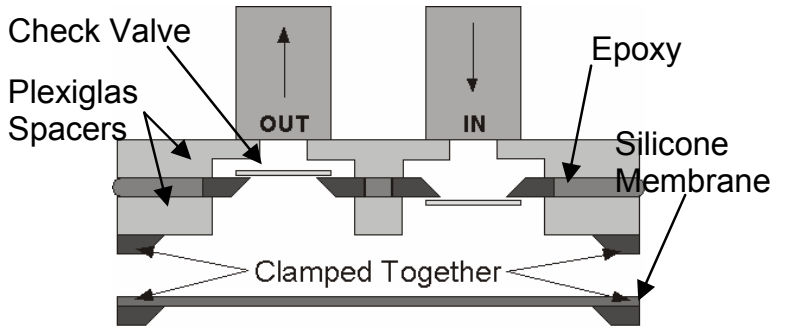

Figure 1: 2D Cross Section of 1st Generation Pump
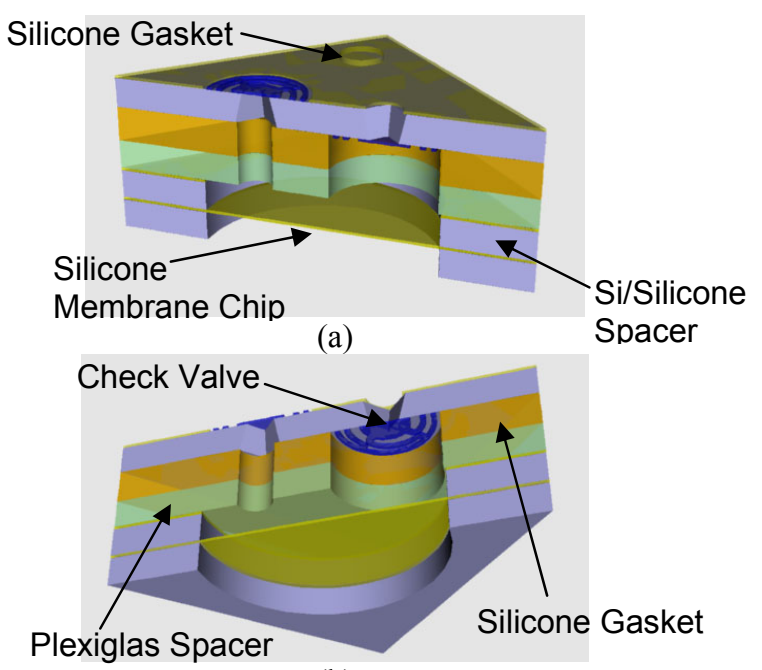

(b)

Figure 2: (a) \& (b) 3D Cross Sections of 2nd Generation Pump 
designed to eliminate the need for glue by taking advantage of the sealing property of silicone rubbers. Alternating layers of silicone and silicon allowed the pump to be watertight when clamped.

\section{Parylene Check Valve}

In the first pump, check valves were fabricated on the same side of the wafer and thus only able to rectify flow in one direction. Pump assembly was complicated by having to dice individual valves and glue them, facing in opposite directions, to a Plexiglas structural support. As a result, the second generation pump check valves were made with a double-sided process shown in Fig. 3.

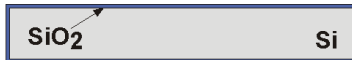

a) Thermal Oxidation

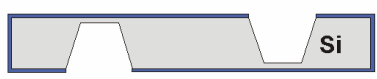

b) KOH Etching

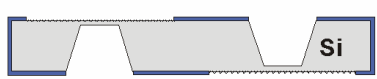

c) $\mathrm{BrF}_{3}$ Roughening + Adhesion Promotion

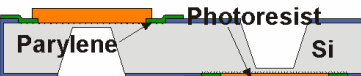

d) 2 um Parylene Deposition + Patterning

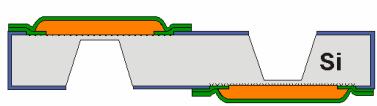

e) Hard Bake PR + $3 \mu \mathrm{m}$ Parylene Deposition

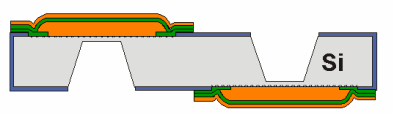

f) Parylene Patterning

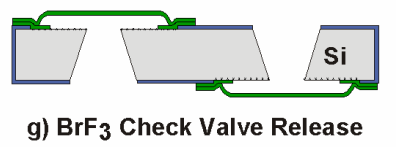

Figure 3: Double-Sided Check Valve Process Flow

The fabrication process started with a thermally oxidized (1.5 $\mu \mathrm{m}$ thick) silicon wafer. Cavities that would eventually become orifices were defined using a $\mathrm{KOH}$ etch. $20 \mu \mathrm{m}$ of silicon was left to serve as a structural support. A circular region of silicon was exposed and roughened using $\mathrm{BrF}_{3}$ gas phase etching. This reduced stiction of the Parylene tethers to the substrate and enhanced adhesion of the anchors. A-174 adhesion promoter was applied followed by the deposition of a $2 \mu \mathrm{m}$ layer of Parylene-C. The Parylene was patterned in an oxygen plasma and then a sacrificial photoresist layer (5 $\mu \mathrm{m}$ of $\mathrm{AZ} 4400)$ was spun and patterned. To prevent check valve failure due to sharp corners in the Parylene, the photoresist was hard baked to round off the convex corners. A second layer of Parylene $(3 \mu \mathrm{m})$ was then deposited and masked with $1000 \AA$ of aluminum during patterning in oxygen plasma. This Al masking layer was replaced with thick photoresist $(10 \mu \mathrm{m}$ of $\mathrm{AZ} 4620)$ in the double-sided process to avoid overheating the photoresist and Parylene layers during thermal evaporation. To release the check valve, the $20 \mu \mathrm{m}$ silicon layer obstructing the orifice was etched away in $\mathrm{BrF}_{3}$. After dicing, the $\mathrm{Al}$ masking layer and sacrificial photoresist layer were removed to complete the fabrication process. Top views of the check valves used

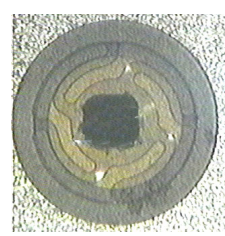

(a)

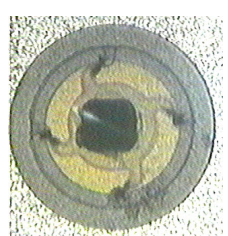

(b)
Figure 4: Check Valves (a) at Rest \& (b) Deflected by Applied Pressure

are shown in Figure 4.

\section{Silicone Membrane}

To efficiently use pump chamber volume, it was necessary to match the chamber geometry with that of the membrane. A large orifice etched into silicon formed a spacer chip that defined the pump chamber when situated between the check valves and pumping membrane. First generation $\mathrm{KOH}$ etched silicone membranes ( $7 \quad 7 \mathrm{~mm}^{2}$ ) and spacers were square making high compression ratios difficult to achieve. By using DRIE, a cylindrical pump chamber and matching circular diaphragm (8 $\mathrm{mm}$ in diameter) could be realized. In addition, it was easier to define cylindrical boss structures without the need for corner compensation.

$20 \mu \mathrm{m}$ silicon membranes are formed via $\mathrm{KOH}$ or DRIE etching. Silicone rubber is spin coated to a thickness of $80 \mu \mathrm{m}$ (first generation) and $140 \mu \mathrm{m}$ (second generation). An $\mathrm{SF}_{6}$ plasma is used to release the

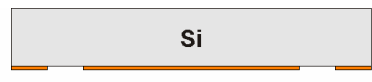

a) Pattern Backside

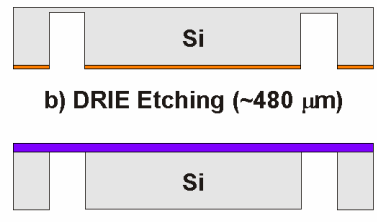

c) Spin Coat $130 \mu \mathrm{m}$ Silicone (MRTV1)

+ Release

Figure 6: Bossed Silicone Membrane Process Flow 


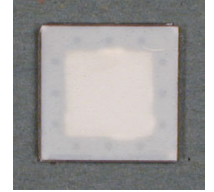

(a)

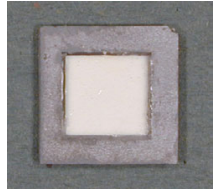

(b)
Figure 7: KOH Etched Silicone Membrane

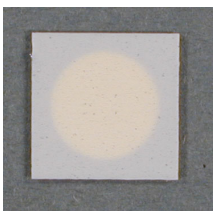

(a)

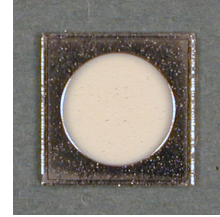

(b)
Figure 8: DRIE Etched Silicone Membrane

membranes. The fabrication process and various membrane designs are shown in Figures 6-8. The first generation spacer is simply an $88 \mathrm{~mm}^{2}$ orifice etched into silicon via $\mathrm{KOH}$. Second generation spacers are formed by removing the membrane portion of the membrane chips. The silicone on the surrounding areas is left as a means to seal the structure during assembly.

\section{Silicone Gasket}

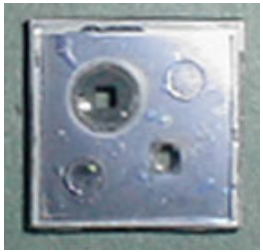

(a)

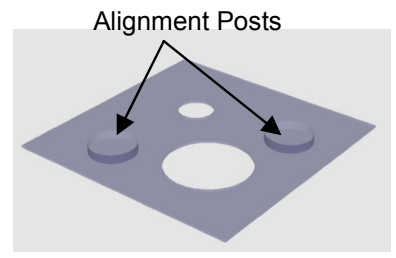

(b)
Figure 9: (a) Gasket on Check Valve Chip \& (b) 3D View of Gasket

Molded silicone gaskets were introduced in the second pump to seal the check valves to the pump seat (Fig. 9). To prevent silicone membranes from adhering to the Parylene check valves during pump operation, it was necessary to insert a machined Plexiglas spacer. However, this created the need for another silicone gasket to seal the spacer to the check valve chip (Fig. 10).

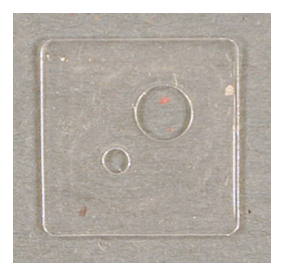

(a)

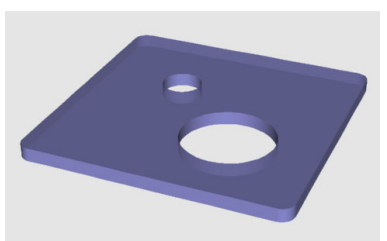

(b)
Figure 10: (a) Gasket \& (b) 3D View of Gasket

Molding silicone into a DRIE etched silicon master (Fig.11) formed the gasket shown in Figure 9. $10 \mu \mathrm{m}$

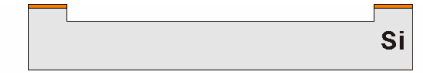

a) Pattern + DRIE Etching ( 100 $\mu \mathrm{m})$

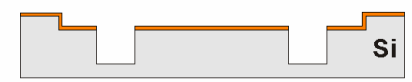

b) Pattern + DRIE Etching ( 300 $\mu \mathrm{m})$

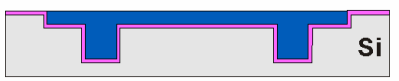

c) Apply Mold Release Layer (Teflon or Parylene) + Squeegee Silicone (Sylgard 184)

Figure 11: Silicon Gasket Process Flow

of photoresist (AZ 4620) was used as a mask for both etching steps. The first etch established the thickness of the gasket $(\sim 100 \mu \mathrm{m})$, and the second produced pits to form alignment pegs $(400 \mu \mathrm{m})$. These pegs mated with pits in a Plexiglas pump seat. A layer of Parylene or plasma deposited Teflon $\left(\mathrm{CHF}_{3}\right)$ can be used as mold release layers. The remaining gaskets were molded from precision milled Delrin masters.

\section{Packaging}

The first generation pump was assembled through a combination of clamping and gluing. The membrane chip was glued to a Plexiglas plate and clamped to the rest of the pump which consisted of chips stacked and glued together. Tygon tubing was connected to the input and output to facilitate testing. A solenoid actuator was then properly positioned beneath the membrane.

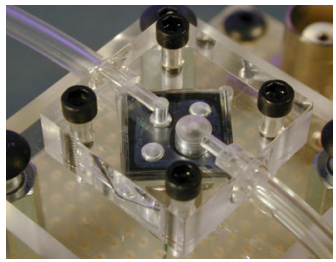

(a)

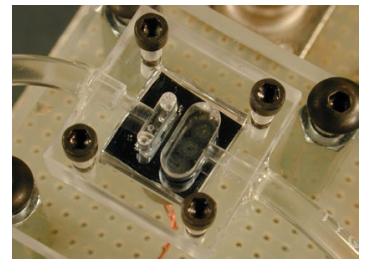

(b)
Figure 13: Top View of (a) Single Inlet/Outlet \& (b) Check Valve Array Pump

The second generation pump (Fig. 13) was entirely clamped and only required adhesive to connect the tubing and actuator to the packaging. This modular design allowed easy access to and replacement of individual components. Two different pump seats were precision milled from Plexiglas for the two different check valve configurations. Plexiglas backing plates allowed the pump to be clamped together and provided a means for attaching the actuator. A different backing plate was used during pneumatic testing. 


\section{TESTING AND RESULTS}

\section{Check Valve}

Performance of the new check valves was characterized for both air and water (Fig. 14). Flow rate versus applied pressure plots with the check valve in place and removed (orifice only) are displayed together. As shown in [12], the twist-up tether configuration of the check valves allowed for enough deflection to achieve nearly atmospheric pressure at the exit. Check valve cracking pressures were below sensor resolution.

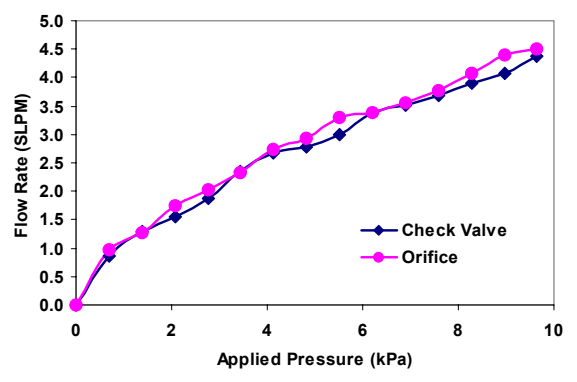

(a)

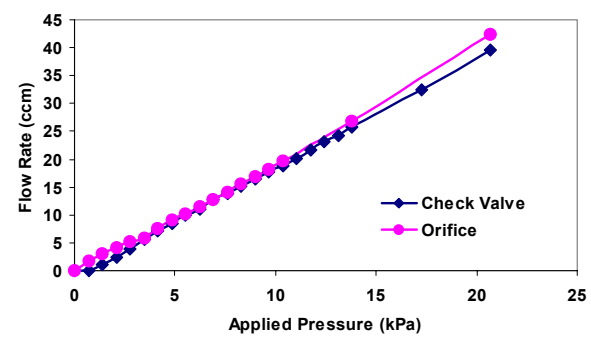

(b)

Figure 14: Flow Rate vs. Applied Pressure Plots for (a) Air \& (b) Water

A novel check valve array, consisting of and inlet and outlet each comprised of three individual check valves, was also characterized. Figure 15 shows the flow rate increase as the number of check valves was increased. Similar data for check valves used in the first generation pump, which have orifice dimensions 780 $\mu \mathrm{m} \quad 780 \mu \mathrm{m}$, was presented in [12]. A slight advantage in performance for water flow was observed in the array case. The orifice dimensions for the solitary input/outlet check valves were $770 \mu \mathrm{m} \quad 770$ $\mu \mathrm{m}$ and for the check valves in the array, $370 \mu \mathrm{m} \quad 370$ $\mu \mathrm{m}$. Effective orifice areas were $5.93 \quad 10^{-3} \mathrm{~mm}^{2}$ and $4.1110^{-3} \mathrm{~mm}^{2}$, respectively. Thus, a check valve array was preferred over a single check valve design to maximize flow rates.

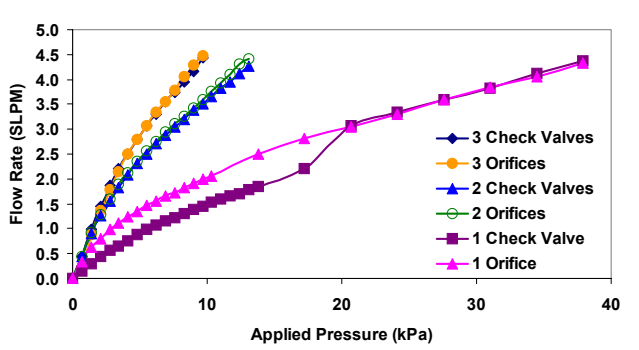

(a)

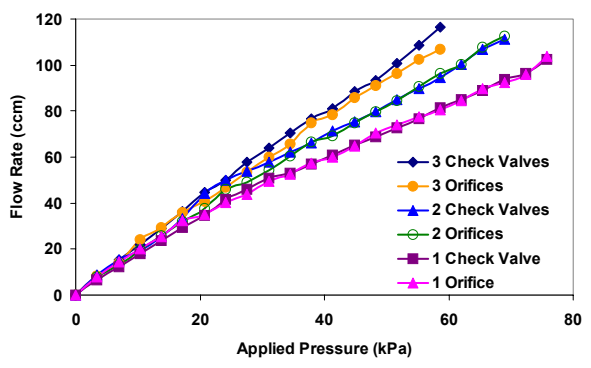

(b)

Figure 15: Flow Rate vs. Pressure in Check Valve Array for (a) Air \& (b) Water

\section{Pump}

\section{$\mathbf{1}^{\text {st }}$ Generation}

The solenoid actuator produced a reciprocating motion where an integrated plunger was retracted and extended

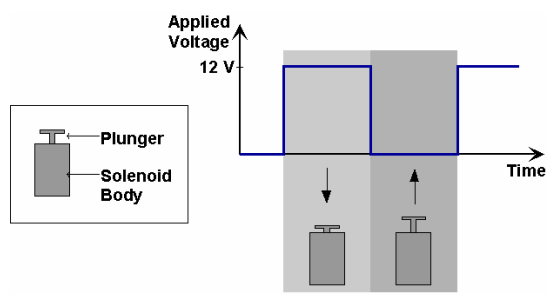

Figure 16: Solenoid Actuation Scheme

as current was turned on and off, respectively (Fig. 16). By using the plunger to deflect the silicone membrane repeatedly, water pumping was achieved. Flow rate versus frequency and back pressure plots are displayed in Figure 17. Careful priming was necessary to achieve optimal performance. As expected, flow rate was highly dependent on actuation frequency and was approximately linear for low frequencies. However, above $10 \mathrm{~Hz}$, this linear relationship was lost as the membrane was unable to match actuator movement. The maximum achievable flow rate was $3.4 \mu \mathrm{l} / \mathrm{min}$ at $10 \mathrm{~Hz}$ and maximum sustainable back pressure was 2.1 $\mathrm{kPa}$ which occurs for both 10 and $20 \mathrm{~Hz}$. 


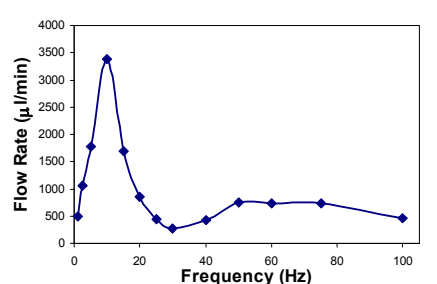

(a)

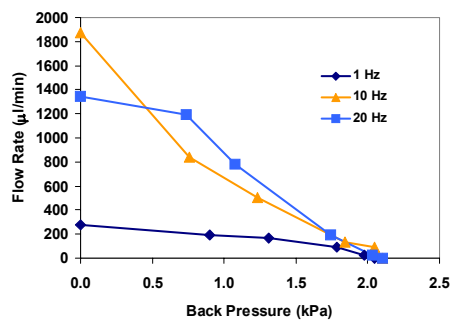

(b)

Figure 17: 1st Generation Pump (a) Flow Rate vs.

Frequency \& (b) Back Pressure Plots

\section{$2^{\text {nd }}$ Generation: Pneumatic Pumping}

Pneumatic operation was performed to verify the design of the second generation pump. Both check valve schemes were tested using an inflation pressure of 6.9 $\mathrm{kPa}$ and the results are shown in Figures 18 and 19. Up to $4.4 \mu \mathrm{l} / \mathrm{min}$ at $13 \mathrm{~Hz}$ and a back pressure of $3.6 \mathrm{kPa}$ at $5 \mathrm{~Hz}$ were possible with the single inlet and outlet design. A nearly threefold increase in flow rate of 13 $\mu \mathrm{l} / \mathrm{min}$ at $11 \mathrm{~Hz}$ and maximum back pressure of $5.9 \mathrm{kPa}$ at $5 \mathrm{~Hz}$ was achieved in the array pump. Thus, the check valve array pump outperformed the single check valve pump in both categories. The same nonlinear behavior in the flow rate dependency on frequency was also observed, occurring for the same reasons as before.

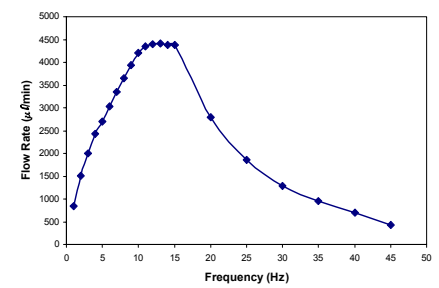

(a)

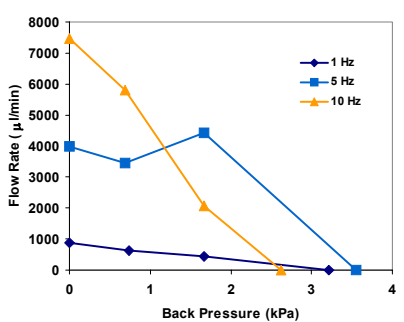

(b)

Figure 18: 2nd Generation Pump with Single Check Valve (a) Flow Rate vs. Frequency \& (b)

Back Pressure Plots for Pneumatic Actuation

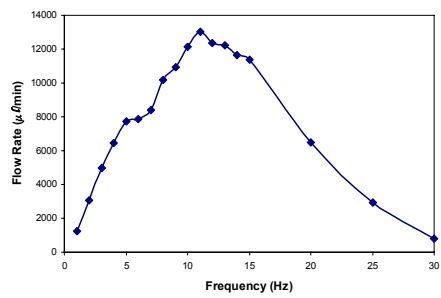

(a)

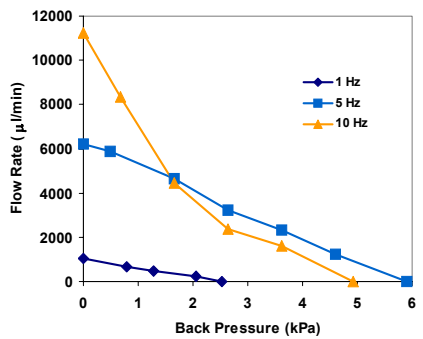

(b)

Figure 19: 2nd Generation Pump with Check Valve Array (a) Flow Rate vs. Frequency \& (b)

Back Pressure Plots for Pneumatic Actuation

\section{$2^{\text {nd }}$ Generation: Solenoid Actuated Pumping}

To eliminate the fall off in flow rate at high frequencies, it was necessary to physically connect the actuator to the pumping membrane. Thus, a new membrane with a circular silicon boss structure was implemented. This allowed the plunger of the actuator to be attached to the pump chamber via a silicon boss on the silicone membrane. The improvement in flow rate versus frequency performance for the single inlet/outlet pump is shown in Fig. 20. A maximum flow rate of 4.5 $\mu \mathrm{l} / \mathrm{min}$ at $14 \mathrm{~Hz}$ was possible. However, the maximum back pressure decreased slightly to $1.32 \mathrm{kPa}$ at 1 and 5 Hz.

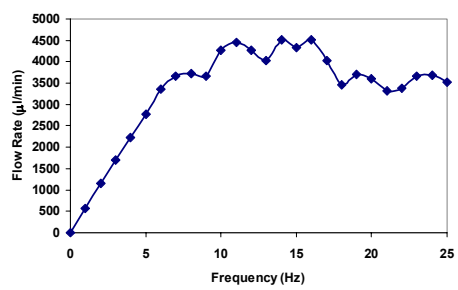

(a)

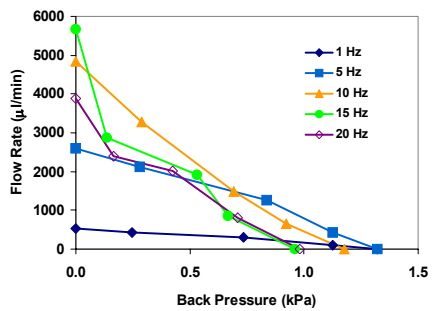

(b)

Figure 20: Solenoid Actuated Pump (a) Flow Rate vs. Frequency \& (b) Back Pressure Plots 


\section{DISCUSSION}

In an ideal situation, the volume of liquid pumped per actuation cycle is a constant regardless of the actuation frequency. However, as can be seen in Figures 21a-d,

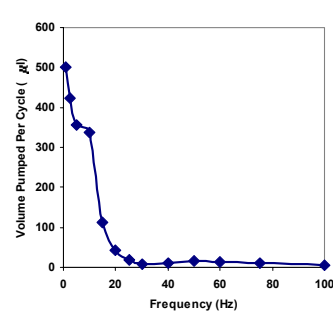

(a)

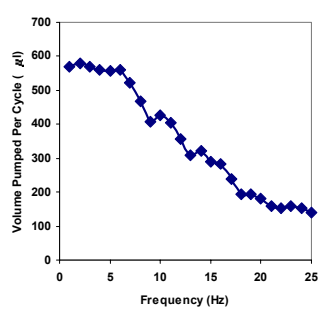

(c)

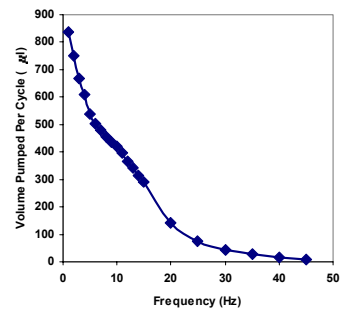

(b)

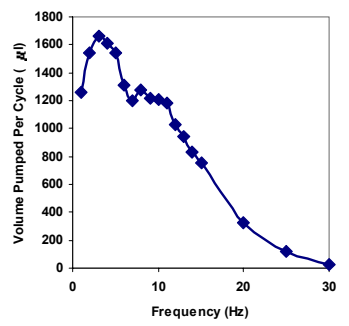

(d)
Figure 21: Volume Pumped Per Cycle vs. Frequency for (a) 1st Generation Pump, 2nd Generation Pump Actuated (b) Pneumatically and (c) with a Solenoid, and (d) 2nd Generation Pump with Check Valve Array Actuated Pneumatically

the volume decreases with increasing frequency. In the pneumatically actuated pump, since flow rate is equal to the pressure drop over the fluidic resistance $(Q=P / R)$, the volume pumped per cycle is inversely proportional to the frequency. Solenoid actuation, in which the membrane and plunger are physically connected, produces a more complicated behavior that cannot be completely described by this simple analysis. The data suggests that the membrane deflection, which occurs in a finite amount of time, might be a contributing factor as it also decreases with increasing frequency.

Discrepancies between the maximum flow rate achieved for pneumatic versus solenoid actuation were due to the inability of the solenoid actuator to produce deflections equal to the height of the pump chamber. For the pumps presented, a solenoid actuator was sufficient to demonstrate the device however, integrated thermopneumatic or piezoelectric actuators are also being investigated.

\section{CONCLUSION}

Two silicone diaphragm pumps are presented. Multiple check valve configurations were explored and a check valve array is optimal for pumping higher volumes. A greater reduction in dead volume and better actuator are necessary to achieve air pumping.

\section{ACKNOWLEDGMENTS}

This work is supported by the DARPA MICROFLUMES program under Naval Ocean Systems Center Contract N66001-96-C-83632. The authors would like to thank Mr. Charles Grosjean and Dr. Qiao Lin for helpful discussions; Mr. Tuan Hoang for help with testing and proofreading; and Dr. Thomas Tsao, Mr. Trevor Roper, and Mr. Hung Bui for assistance with fabrication.

\section{REFERENCES}

[1] van Lintel, H.T.G., F.C.M. van de Pol, and S. Bouwstra, A Piezoelectric Micropump Based on Micromachining of Silicon. Sensors and Actuators A, 1988. 15: p. 153-167.

[2] van de Pol, F.C.M., et al., A Thermopneumatic Micropump Based on Micro-engineering Techniques. Sensors and Actuators A, 1990. 21(13): p. 198-202.

[3] Zengerle, R., A. Richter, and H. Sandmaier, A Micro Membrane Pump With Electrostatic Actuation. MEMS '92, 1992: p. 19-24.

[4] Olsson, A., et al., An Improved Valve-less Pump Fabricated Using Deep Reactive Ion Etching, in MEMS '96. 1996. p. 479-484.

[5] Jiang, X.N., et al., Micronozzle/diffuser flow and its application in micro valveless pumps. Sensors and Actuators A, 1998. 70: p. 81-87.

[6] Fuhr, G., et al., Pump of Water Solutions in Microfabricated Electrohydrodynamic Systems. MEMS '92, 1992: p. 25-30.

[7] Ahn, S.-H. and Y.-K. Kim, Fabrication and experiment of a planar micro ion drag pump. Sensors and Actuators A, 1998. 70: p. 1-5.

[8] Ahn, C.H. and M.G. Allen, Fluid Micropumps Based on Rotary Magnetic Actuators, in MEMS '95. 1995. p. 408-12.

[9] Döpper, J., et al., Micro gear pumps for dosing of viscous fluids. Journal of Micromechanics and Microengineering, 1997. 7: p. 230-2.

[10] Shoji, S., et al., A study of a high-pressure micropump for integrated chemical analysing systems. Sensors and Actuators A, 1992. 32: p. 335-339.

[11] Smith, L. and B. Hök, A Silicon Self-Aligned NonReverse Valve. Transducers '91, 1991: p. 10491051.

[12] Wang, X.-Q., Q. Lin, and Y.-C. Tai, A Parylene Micro Check Valve, in MEMS '99. 1999. p. 177182.

[13] Yang, X., C. Grosjean, and Y.-C. Tai, A low power MEMS silicone/parylene valve, in 1998 Solid State Sensor and Actuator Workshop. 1998. p. 316-9.

[14] Grosjean, C. and Y.-C. Tai, A Thermopneumatic Peristaltic Micropump. Transducers '99, 1999. 